\title{
INVESTIGATION OF GALACTIC STRUCTURE WITH DENIS STAR COUNTS
}

\section{S. RUPHY}

\author{
Observatoire de Paris
}

\section{Introduction}

Thanks to the DENIS program (Deep Near-Infrared Survey of the Southern Sky), relatively deep near-infrared star counts are now available for the first time on a large scale. The basic method to interpret star counts in terms of galactic structure is to compare them with predictions given by models of the point source sky. Of particular promise are studies with DENIS of the spatial distribution of evolved stars in our Galaxy, thanks to its high sensitivity to red giant and to the much lower interstellar extinction that hampers visual observations of far-away stars in the disc of our Galaxy. In this paper, I present a sample of extensive comparisons between two models of the Galaxy and DENIS star counts (Ruphy 1996). I will focus on the analysis of star counts in the anticenter direction, that leads to new values for the distance of the cutoff and the radial scale length of the stellar disc.

\section{Observations}

The observations presented here are part of the first data released by the DENIS survey which will provide, before the end of the century, the first digitized survey of the southern sky, with a $3 \sigma$ detection limit of 18,16 and 14 , in the I $(0.8 \mu \mathrm{m}), \mathrm{J}(1.25 \mu \mathrm{m})$ and $K_{s}(2.15 \mu \mathrm{m})$ bands respectively (Epchtein, this volume, p. 106). The observations were made in February and November 1995, using the DENIS camera attached at the focus of the ESO $1 \mathrm{~m}$ telescope at La Silla, Chile. 


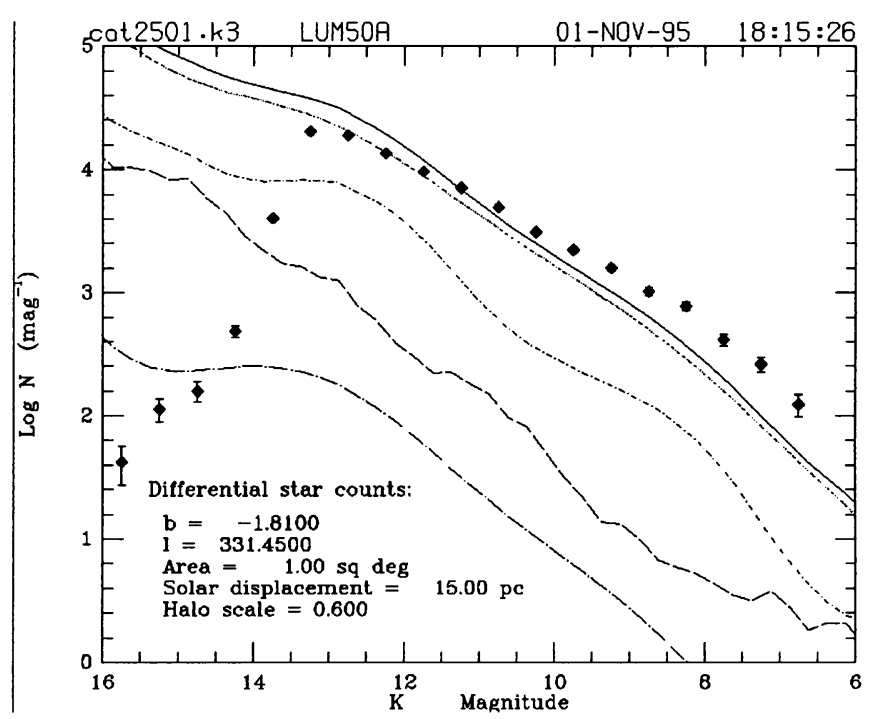

Figure 1. Comparison of SKY predictions and DENIS differential star counts in $K_{s}$, at $\mathrm{l}=331^{\circ}, \mathrm{b}=-1.81^{\circ}$, on $1 \mathrm{deg}^{2}$. Diamonds, observed star counts with poissonian error bars; solid line, total model prediction; dots, disc; long dashes, spiral arms; long dash-dot, halo; short dash-dot, ring.

\section{Comparisons with Models of the Point Source Sky}

\subsection{STUDY OF THE INHOMOGENEITIES OF THE GALACTIC PLANE WITH THE 'SKY' MODEL}

The SKY model is based on a realistic representation of the Galaxy, including features such as disc, spiral arms, local spur, molecular ring, bulge and halo, and delivers star counts in many filters lying within a large spectral range, from far-UV to far-infrared. The basic model is described by Wainscoat et al. (1992), and was considerably enhanced by Cohen (1994a,b). Comparisons with DENIS star counts in $\mathrm{J}$ and $\mathrm{K}$ at different longitudes along the galactic plane, such as the one presented in Figure 1, allow to improve the description of the spiral arms and the molecular ring in SKY : the lack of bright stars in the model is likely to be due to an inaccurate luminosity function of the spiral arms, and the excess of faint stars can be reduced by changing the geometry of the molecular ring and making it elliptical (Ruphy 1996).

\subsection{NEW DETERMINATION OF THE DISC SCALE LENGTH AND RADIAL CUTOFF IN THE ANTICENTER WITH THE BESANÇON MODEL}

Comparisons in the anticenter direction have revealed a systematic excess of stars predicted by both SKY and the Besançon model. The Besançon 
model is a synthetic stellar population model developped by Robin \& Crézé (1986). In the outer part of the galactic plane, the most influential parameters are the disc scale length $h_{R}$ and the distance of the edge of the disc $R_{\text {max }}$. We compared J-K colour distributions in two strips (a strip covers $\simeq 5.75 \mathrm{deg}^{2}$ ) crossing the galactic plane at $l=217^{\circ}$ and $1=239^{\circ}$ respectively, with predictions given by the Besançon model for a large range of $h_{R}$ and $R_{\max }$ values. The best values are obtained by using a maximum likelihood method applied on a set of bins of $\mathrm{K}$ and $\mathrm{J}-\mathrm{K}$, in different declination ranges (see Ruphy et al. 1996, for details of the procedure and further discussions). For the two strips, the maximum likelihood is obtained for the same values, namely $R_{\max }=15 \pm 2 \mathrm{kpc}$ and $h_{R}=2.3 \pm 0.1 \mathrm{kpc}$.

\section{Conclusion}

Thanks to the very first DENIS data, we confirmed the existence of the cutoff in our Galaxy and obtained a rather short scale length for the disc. Our value is compatible with previous determinations based on star counts in the visible, in the anticenter direction (Robin et al. 1992) or in central parts of the Galaxy (Ojha et al. 1996). It is also in agreement with the recent value found by Fux \& Martinet (1994) from kinematical data. Our determination benefits from a small influence of the extinction and the large size of the samples ( $\simeq 16,000$ stars per strip). Furthermore, it is based not only on the dwarf population, as it is the case for analysis of star counts in the visible, but also on the giant population.

\section{Acknowledgements}

I thank M. Cohen and A. C. Robin for their close collaboration in the interpretation of the DENIS counts with their models. The DENIS team is also warmly thanked for making the observations available for scientific analysis.

\section{References}

Cohen M. 1994a, Astron.J., 107, 582

Cohen M. 1994b, Astrophys.J., 427, 848

Fux R., Martinet L., 1994, Astron.Astrophys., 287, L21

Ojha D.K, Bienaymé O., Robin A.C., Crézé M., Mohan V., 1996, Astron.Astrophys., 311,456

Robin A. C. and Crézé M., 1986, Astron.Astrophys., 157, 71

Robin A. C., Crézé M., Mohan V., 1992, Astrophys.J., 400, L25

Ruphy S., Robin A. C., N. Epchtein, Copet E., Bertin E., Fouqué P., Guglielmo F., 1996, Astron.Astrophys., 313, L21

Ruphy S., 1996, PhD. dissertation, Paris

Wainscoat R. J., Cohen M., Volk K., Walker H. J., Schwartz D E., 1992, Astrophys.J.Suppl., 83, 111 